\title{
Morphological stability of a heterophase interface under electromigration conditions
}

\author{
L. Klinger and L. Levin \\ Department of Materials Engineering, Technion-Israel Institute of Technology, Haifa, Israel \\ D. Srolovitz ${ }^{\text {a) }}$ \\ Department of Materials Science and Engineering, University of Michigan, Ann Arbor, Michigan 48104
}

(Received 28 November 1995; accepted for publication 8 January 1996)

\begin{abstract}
The evolution of the interface between two mutually insoluble metallic phases, under the influence of a strong electric field is examined. A slight perturbation of the interface away from a plane $y=h(x)$ leads to a component of the electric field along the interface. This creates a diffusion flux of the individual atoms along the interface which, in turn, leads to an increase in the amplitude of the initial perturbation and thus to an interfacial profile instability. The processes is expected to be controlled by interface diffusion in response to three distinct driving forces: the electric field, internal stresses (which arise due to the accumulation or depletion of matter at the interface), and the interfacial curvature. The stress distribution along the interface was found from a self-consistent solution of the elastic problem. For the instability to occur, differences in effective atomic charges, elastic moduli and/or atomic mobilities of the two constituent metals are required. Small sinusoidal corrugations are shown to grow with time for a range of wavelengths. The corrugations can grow monotonically or vary in oscillatory manner, depending on their wavelength. (C) 1996 American Institute of Physics. [S0021-8979(96)06608-3]
\end{abstract}

\section{INTRODUCTION}

Instabilities of planar interfaces have been investigated in many different contexts, including solidification, ${ }^{1}$ chemical reactions, ${ }^{2,3}$ and mechanical deformation. ${ }^{4-7}$ The present work focuses on the morphological instability of an initially planar heterophase interface in a strong, perpendicular electric field. A related instability has been observed at a heterophase interfaces in an ionic system. ${ }^{8}$ This strong electric field, commonly causes an electric current. In metallic systems, the resulting electron current interacts with the atoms in the solid causing an atomic flux known as electromigration. This atomic flux will be discontinuous across an interface between two materials provided that the effective charge on the atoms or the atomic mobility in the two materials is different. This discontinuity can lead to the destabilization of such an initially flat heterophase interface. A steady state analysis of this problem was presented in Ref. 9.

Instabilities in interfacial morphology generally require either material transport or a phase change. Focusing on the former situation, the interface velocity is controlled by the relatives rates at which matter is attached to the phases meeting at the interface. In the case of fluid-fluid or solid-fluid interfaces, this addition of material volume at the interface causes fluid flow. At solid-solid interfaces, on the other hand, this material accumulation or depletion at the interface can set up stresses within the solids that create a reverse driving force that opposes the initial atomic flux. Typically, this is accounted for in interfacial problems by postulating that an accumulation of material on one side of the interface is accompanied by depletion of material from the opposing side of the interface such that there is no net material accumulation or depletion. In the present paper, we relax this

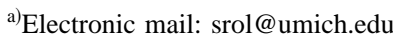

assumption by explicitly accounting for the evolution of stresses within the material and coupling these stresses back to the material flux.

In the present work, we perform a self-consistent analysis of the morphological stability of an initially flat heterophase interface separating from two immiscible solids in an orthogonal electric field evolving under the action of interfacial diffusion. We consider electromigration, the Kirkendall effect (since the atomic mobilities in the two materials can be different), and the case of arbitrary effective charge on the atoms. Using this approach, we derive the conditions under which such an interface will be unstable, predict the rate at which this instability develops, and predict the wavelength that is expected to grow the fastest.

\section{INTERFACE DIFFUSIONAL FLUX}

The interaction of metal ions with the "electron wind" causes a diffusion flux of the atoms along (or opposite to) the electron current. At high temperatures this can lead to bulk atomic transport, while at low and intermediate temperatures diffusion occurs primarily along grain boundaries, interfaces, and/or surfaces. ${ }^{10}$ The present work focuses on an initially planer interface at $y=0$ between two distinct metallic phases $\alpha-\beta$, composed of mutually insoluble atomic species $A$ and $B$, respectively. The electric current density $j$ is oriented perpendicular to the initially flat interface (see Fig. 1). For simplicity, we assume that matter transport is diffusive and occurs solely along this heterophase interface. The electric field does not affect the interface when it is planar $(y=0)$, however, any perturbation to the flat surface $y=h(x)$ results in a component of the electric field along the interface. This, in turn, causes a diffusion flux of the chemical components 


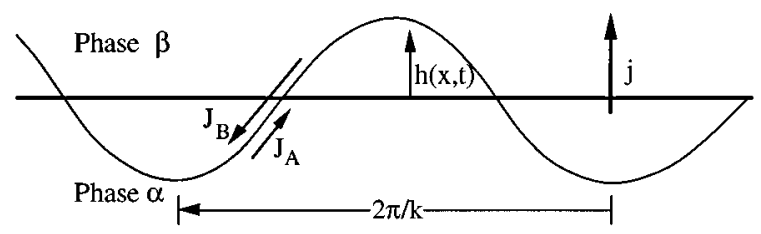

FIG. 1. Schematic illustration of a sinusoidal perturbation (of wave number $k$ ) to an initially flat interface separating phases $\alpha$ and $\beta$. The surface profile amplitude $h(x, t)$ evolves under the action of the flux of $A$ and $B$ atoms, $J_{A}$ and $J_{B}$, respectively, which are driven by the electric current flux $j$.

along the interface, which may increase the amplitude of the interface perturbation (see Fig. 1) and thereby lead to an instability in the interface morphology.

The volume diffusional flux (i.e., the volume of atoms passing through a unit length of boundary per unit time) along the interface is proportional to the gradient of the chemical potential along the interface

$$
J_{\eta}=-\frac{\delta D_{\eta}}{k T} \nabla_{i} \mu_{\eta} \quad(\eta=A, B)
$$

where $D_{\eta}$ is the interface diffusion coefficient of atomic component $\eta, \delta$ is the diffusional width of the interface, $k$ is Boltzmann's constant, $T$ is the temperature, and $\nabla_{i} \mu_{\eta}$ is the gradient of the chemical potential of atomic component $\eta$ along the interface. We consider three distinct causes for the change of the chemical potentials along the interface

$$
\mu_{\eta}-\mu_{\eta}^{0}=z_{\eta} \varphi+\omega_{\eta} \sigma \mp \omega_{\eta} \gamma \kappa \quad(\eta=A, B)
$$

where $\mu_{\eta}^{0}$ is the chemical potential of the flat, stress free interface in zero electric field. The first term on the righthand side of Eq. (2) is the chemical potential change due to the electric field $\left(z_{A}\right.$ and $z_{B}$ are the effective atomic charges, $\varphi$ is the electric potential). A divergence of the diffusion fluxes at the interface will lead to the accumulation or depletion of matter there and thus produce concomitant internal stresses. The influence of these stresses on the chemical potential is expressed by the second term in Eq. (2), where $\omega_{A}$ and $\omega_{B}$ are the atomic volumes associated with $A$ and $B$ atoms, and $\sigma$ is the normal stress acting across the interface. The third contribution to the chemical potential along the interface is associated with the curvature of the interface in a manner akin to its affect at gas-solid interfaces. ${ }^{11} \gamma$ is the interfacial energy per unit area and $\kappa$ is the interface curvature. The sign of the curvature term in Eq. (2) is opposite for the materials on either side of the interface (in phases $\alpha$ and $\beta$ ). For the $\alpha$ phase, the " - ", sign is chosen and for the $\beta$ phase, we chose the " + " sign. Substituting Eq. (2) into Eq. (1) yields

$$
J_{\eta}=-L_{\eta}\left( \pm \gamma \boldsymbol{\nabla}_{i} \kappa+q_{\eta} \boldsymbol{\nabla}_{i} \varphi+\nabla_{i} \sigma\right) \quad(\eta=A, B)
$$

where $L_{\eta}=\delta D_{\eta} \omega_{\eta} / k T$ is the volume mobility, $D_{\eta}$ is the diffusivity of atomic component $\eta$ at the interface and $q_{\eta}=z_{\eta} / \omega_{\eta}$ is the volume charge of atomic component $\eta$. If the volume fluxes of components $A$ and $B$ are equal and opposite, then no matter is accumulated or depleted from the interface and hence no stresses develop. In general, however, a divergence of the total diffusion flux $\left(J_{A}+J_{B}\right)$ leads to accumulation or depletion of matter at the interface and the concomitant development of stress. Therefore, the stress distribution must be determined self consistently with respect to the atomic fluxes.

We can determine the elastic state of the interface in terms of the accumulation-depletion of matter at the interface and the overall motion of the interface itself. The relationship between the stresses, atomic fluxes, and boundary positions are discussed in detail in Ref. 12. If the phases on either side of the interphase boundary were decoupled (or separated), then the displacement of the surface (one side of the boundary) at any point would simply be related to the divergence of the volume interfacial flux at that point. In the case of an interface, however, the two surfaces are joined. This joining operation displaces the interface from its original position such that the interface lies at $y=h$ instead of $y=0$. The elastic displacements (i.e., those that produce stresses) at the interface is simply the difference between the displacement of the decoupled boundary due to matter accumulation-depletion and the displacement of the joined interphase boundary. Therefore, the elastic displacement of the two phases $u_{A}$ and $u_{B}$ evaluated at the interface can be written as

$$
\begin{aligned}
& \frac{\partial u_{A}}{\partial t}=-\frac{\partial J_{A}}{\partial x}-\frac{\partial h}{\partial t} \\
& \frac{\partial u_{B}}{\partial t}=-\frac{\partial J_{B}}{\partial x}+\frac{\partial h}{\partial t}
\end{aligned}
$$

where $h(x, t)$ is the interface profile. Introducing $u=u_{A}+u_{B}$, Eqs. (4) can be rewritten as

$$
\begin{aligned}
& \frac{\partial u}{\partial t}=-\frac{\partial}{\partial x}\left(J_{A}+J_{B}\right) \\
& \frac{\partial h}{\partial t}=-\frac{\partial}{\partial x}\left[\xi J_{A}-(1-\xi) J_{B}\right]
\end{aligned}
$$

where $\xi=u_{B} /\left(u_{A}+u_{B}\right)$. In the Appendix (Eq. A13), we show that $\xi$ can also be written as

$$
\xi=\frac{G_{A}}{\left(G_{A}+G_{B}\right)}
$$

where $G_{A}$ and $G_{B}$ are elastic moduli of the $A$ and $B$ phases defined in the Appendix, respectively.

\section{INTERFACE EVOLUTION}

We now analyze the temporal evolution of a small, sinusoidal perturbation to the interface profile of wavelength $2 \pi / k$ :

$$
h(x, t)=H(t) \sin (k x)
$$

where $H k \ll 1$. In this small slope limit, the fluxes, elastic displacements and interface profile are periodic with the same period. The gradient of the curvature is $\boldsymbol{\nabla}_{i} \kappa=-k^{3} H \cos (k x)$. The electric field gradient is 9

$$
\nabla_{i} \varphi=-k F H \cos (k x)
$$


where $F=2 j \rho_{A} \rho_{B} /\left(\rho_{A}+\rho_{B}\right) ; \rho_{A}$ and $\rho_{B}$ are the specific electric resistivities of the phases, $\alpha$ and $\beta$. Denoting the amplitude of the elastic displacement $u$ as $U(t)$ yields

$$
u(x, t)=U(t) \sin (k x) .
$$

The stress normal to the interface $\sigma$ can be determined from the solution of the relevant elastic problem, as described in the Appendix,

$$
\sigma(x, t)=G k U \sin (k x)
$$

where $G$ is a function of the elastic moduli of the $\alpha$ and $\beta$ phases (see the Appendix). Substituting Eqs. (6)-(9) into Eqs. (3)-(5) yields a set of two linear differential equations for the amplitudes of the surface profile $H(t)$ and the elastic displacements at the interface $U(t)$

$$
\begin{aligned}
& \frac{d H}{d t}=a_{11} H+a_{12} U, \\
& \frac{d U}{d t}=a_{21} H+a_{22} U
\end{aligned}
$$

where the coefficients of the matrix, $a_{i j}$, are

$$
\begin{aligned}
a_{11}= & -k^{4} \gamma\left[\xi L_{A}+(1-\xi) L_{B}\right]-k^{2}\left[(1-\xi) q_{B} L_{B}\right. \\
& \left.-\xi q_{A} L_{A}\right] F, \\
a_{12}= & k^{3} G\left[(1-\xi) L_{B}-\xi L_{A}\right], \\
a_{21}= & k^{4} \gamma\left(L_{B}-L_{A}\right)+k^{2}\left(q_{B} L_{B}+q_{A} L_{A}\right) F, \\
a_{22}= & -k^{3} G\left(L_{A}+L_{B}\right) .
\end{aligned}
$$

The temporal evolution of the interface profile and the stresses can be determined by solving Eq. (10). The general solution to Eqs. (10) is

$$
\begin{aligned}
& H(t)=H(0)\left[a e^{p_{+} t}+(1-a) e^{p_{-} t}\right] \\
& U(t)=U(0)\left[b e^{p_{+} t}+(1-b) e^{p_{-} t}\right]
\end{aligned}
$$

where $\{a, b\}$ is the eigenvector and $p_{-}$and $p_{+}$are the characteristic values of matrix $a_{i j}$. The characteristic values are

$p_{ \pm}=\frac{a_{11}+a_{22}}{2} \pm \sqrt{\left(\frac{a_{11}+a_{22}}{2}\right)^{2}-\left(a_{11} a_{22}-a_{12} a_{21}\right)}$.

The flat $(H=0)$ and unstressed $(U=0)$ interface is stable provided that the determinant of the matrix $a_{i j}$ is positive and the trace of the matrix $a_{i j}$ is negative, i.e.,

$$
a_{11} a_{22}-a_{12} a_{21}>0 \text { and } a_{11}+a_{22}<0 .
$$

Inserting the expressions for the coefficients $a_{i j}$ from Eq. (11) into these expressions yields

$$
\begin{aligned}
a_{11} a_{22}- & a_{12} a_{21}=k^{5} L_{A} L_{B} G\left[2 \gamma k^{2}+\left(q_{B}-q_{A}\right) F\right]>0, \\
a_{11}+a_{22}= & -k^{2}\left[\left(\xi L_{A}+(1-\xi) L_{B}\right)\left(\gamma k^{2}+2 G k\right)\right. \\
& \left.+\left((1-\xi) q_{B} L_{B}-\xi q_{A} L_{A}\right) F\right]<0 .
\end{aligned}
$$

Examination of expressions (15a) and (5c) show that the interphase boundary will be stable against perturbations for any wave number provided that

$$
q_{B} \geqslant q_{A} \quad \text { and } G_{B} q_{B} L_{B} \geqslant G_{A} q_{A} L_{A} .
$$
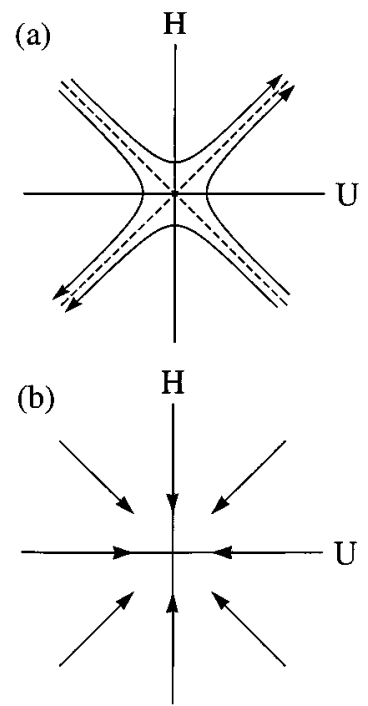

FIG. 2. Schematic illustration of the temporal evolution of the amplitudes of the interface profile perturbation $H$ and the interface elastic displacement $U$ due solely to the differences in atomic charges, as indicated by the arrows, for (a) $k>k_{c}$ and for (b) $k<k_{c}$.

Note that the asymmetry in these results with respect to the two phases is associated with the vector nature of the electric current (see Fig. 1). If, however, either one or both of these conditions (16) are not satisfied, then ranges of wavelengths exist for which perturbations to the interface profile will grow (i.e., the flat interface is unstable). The instability requires either a difference in atomic charges $\left(q_{B}<q_{A}\right)$, a difference in the atomic mobilities $\left(L_{B}<L_{A}\right)$, or a difference in elastic moduli $\left(G_{B}<G_{A}\right)$. We analyze these cases below.

\section{A. Instability due to the difference in the atomic charges: $q_{A}-q_{B} \equiv \Delta q>0 ; L_{B}=L_{A} \equiv L$}

As follows from Eq. (15a), the range of wave numbers $k$ over which the interface is unstable is

$$
k<\sqrt{\Delta q F / 2 \gamma} .
$$

If $G \gg \sqrt{\gamma \Delta q F}$, as is common, Eq. (15b) can be simplified and the characteristic values of the matrix $a_{i j}, p_{ \pm}$are found to be

$$
p_{+}=k^{2} L\left(\Delta q F-2 \gamma k^{2}\right)
$$

and

$$
p_{-}=-k^{3} G L \text {. }
$$

The rate of the growth of the interface profile perturbation is controlled by $p_{+}$, since $p_{-}$is negative.

This analysis shows that interface profile perturbations with wave numbers greater than $k_{c}=\sqrt{\Delta q F / 2 \gamma}$ will decay and those with $k<k_{c}$ will grow. Similarly, the amplitude of the stresses at the interface will grow for $k<k_{c}$ and otherwise decay. In other words, the stresses and the profile perturbation both grow or both decay, as indicated in Fig. 2(a) and (b), respectively. The rate at which the profile and stress 
amplitudes grow for $k<k_{c}$ initially increase with $k$ as $k^{2}$ and decay for large $k$ as $k^{4}$. The perturbation which grows the fastest has a wave number $k_{m}=\sqrt{\Delta q F / 4 \gamma}$.

This same result was previously obtained in an analysis that explicitly assumes that there is no net matter accumulation or depletion anywhere on the interface. ${ }^{9}$ Indeed, it follows from the condition $J_{A}+J_{B}=0$ and that $a_{21} H+a_{22} U=0$. Substituting the latter expression into Eq. (10a) yields

$$
\frac{d H}{d t}=p_{+} H
$$

where $p_{+}$is defined in Eq. (18a).

\section{B. Instability due to the difference in the mobilities and/or the elastic moduli: $q_{B}=q_{A} \equiv q$ and $\left(G_{A} L_{A}-G_{B} L_{B}\right) /\left(G_{A} L_{A}+G_{B} L_{B}\right) \equiv \Delta>0$}

We now examine the case where the effective charges of the two components are equal and show that even in this case, the interface can still be unstable (i.e., will not remain flat), depending on the relative magnitudes of the atomic mobilities and the elastic moduli of the two phases. The conditions for interface stability were given in Eq. (14). In the present case, where $a_{11} a_{22}-a_{12} a_{21}=2 k^{7} L_{A} L_{B} G>0$, we find that the interface is unstable provided that $a_{11}+a_{22}>0$. Using Eq. (15b), we find that the interface is unstable provided that

$$
k<k_{1}=-G / \gamma+\sqrt{(G / \gamma)^{2}+q F \Delta / \gamma} .
$$

In the limit that $G \gg \sqrt{\gamma q F \Delta}$, Eq. (20) simplifies to

$$
k<k_{1} \approx \frac{q F \Delta}{2 G} .
$$

In terms of this definition of $k_{1}$, we can rewrite the expression for the characteristic values of the matrix $a_{i j}$ [see Eq. (13)] as

$$
p_{ \pm}=k^{2} L G\left(\left(k_{1}-k\right) \pm \sqrt{\left(k_{1}-k\right)^{2}-\theta k^{3} \gamma / G}\right)
$$

where $L=\left[\xi L_{B}+(1-\xi) L_{A}\right] / 2, \quad$ and $\quad \theta=2 L_{A} L_{B} /\left[\xi L_{B}\right.$ $\left.+(1-\xi) L_{A}\right]^{2}$.

We now examine the structure of the characteristic values of $a_{i j}$. The expression under the square root in Eq. (22) is positive at small $k$, implying that the interface is unstable against perturbations of small $k$ or large wavelength. If $\theta k^{3} \gamma / G>\left(k_{1}-k\right)^{2}$, then the characteristic values $p_{ \pm}$are complex. Expanding Eq. (22) about $k$ near $k_{1}$, we find that $p_{ \pm}$is complex in the range of $k_{1}-\delta k<k<k_{1}+\delta k$, where

$$
\delta k \approx k_{1} \sqrt{\frac{\alpha k_{1} \gamma}{G}} \ll k_{1} .
$$

We are now in a position to analyze the temporal behavior of the interface profile and the stresses. There are four distinct types of behavior in the $q_{A}=q_{B}$ and $\Delta>0$ case:

\section{1. $k<k_{1}-\delta k$}

In this interval, $p_{+}$and $p_{-}$are both real and positive. This implies that the magnitude of both the stress and the interface perturbation increase with time, as indicated in Fig. 3(a). The actual sign of each depends on the initial condi-
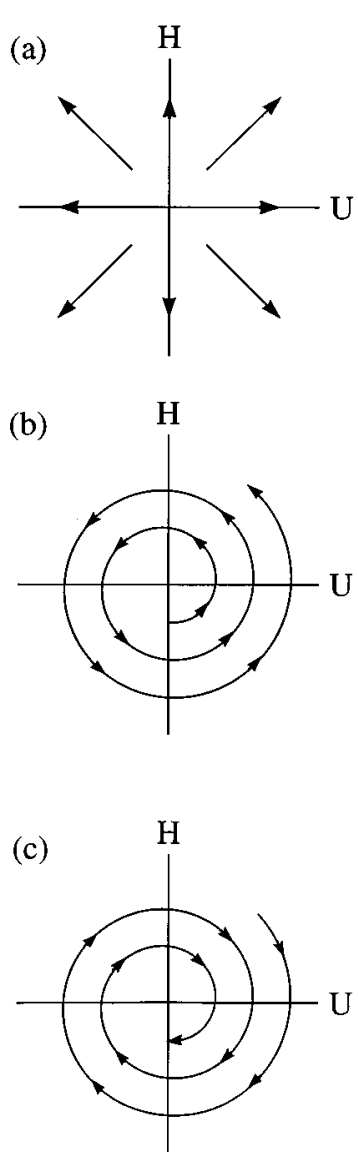

FIG. 3. Schematic illustration of the temporal evolution of the amplitudes of the interface profile perturbation $H$ and the interface elastic displacement $U$ due to differences in atomic mobilities and/or the elastic moduli of phases $\alpha$ and $\beta$, as indicated by the arrows. (a) corresponds to $k<k_{1}-\delta k$, (b) to $k_{1}-\delta k<k<k_{1}$, and (c) to $k_{1}<k<k_{1}+\delta k$ and the case in which $k_{1}+\delta k<k$ is identical with that shown in Fig. 2(b).

tions. The perturbation with $k=2 k_{1} / 3$ grows at the maximum rate and, therefore, will likely be the perturbation wave number that is observed.

\section{2. $k_{1}-\delta k<k<k_{1}$}

In this interval, $p_{+}$and $p_{-}$are complex and the real parts are equal and positive. This implies the amplitudes of the stress and the interface perturbation oscillate and grow with time, as shown in Fig. 3(b). The period of these oscillations $\tau$ can be estimated from Eq. (22) as follows:

$$
\tau \approx \frac{2 \pi}{L k_{1} \sqrt{\theta k_{1} \gamma G}} .
$$

\section{3. $k_{1}<k<k_{1}+\delta k$}

In this interval, $p_{+}$and $p_{-}$are complex and the real parts are equal and negative. This implies the amplitudes of the stress and the interface perturbation oscillate and decay with time, as shown in Fig. 3(c). The period of these oscillations is $\tau$. 


\section{4. $k_{1}+\delta k<k$}

In this interval, $p_{+}$and $p_{-}$are both real and negative. This implies that the magnitude of both the stress and the interface perturbation decrease with time without oscillation. This is the same type of decay of both the stress and interface profile amplitude as was shown in Fig. 2(b).

These results suggest that differences in mobilities and/or moduli of the two phases meeting at the boundary can produce a rich variety of morphological phenomena. We are unaware of any experimental examples in which this type of oscillatory phenomena have been observed, however, we hope that these results will inspire new experiments on electromigration across heterophase interfaces.

\section{CONCLUSION}

A planar interface separating two materials may be unstable in an electric field which has a component normal to the interface. We have considered the case of the growth of an initially small amplitude sinusoidal perturbation to the interface profile, where matter transport occurs by interface diffusion. This instability has two distinct physical origins, either of which is sufficient to destabilize the interface. These are associated with (1) the difference in the effective charges on the atoms in the two materials and (2) the difference in the atomic mobilities or elastic moduli of the two materials. Each of these phenomena can lead to a build up of stress at the interface as a result of the net flux of atoms into or out of the interface. Such stresses can be relieved by the development of interfacial corrugations, much in the same way as occurs for the interface or surface of any stressed solid. ${ }^{4,5}$ The present analysis predicts the wavelengths of the interface corrugations which are expected to grow and identifies the wavelength that should grow the fastest. The wavelengths at which this instability occurs decreases with increasing electric field, effective charge difference, atomic mobility difference, and decreasing interfacial energy. When the atomic mobilities or elastic moduli of the two phases differ, a range of wavelengths exist for which the amplitudes of both the stress and the interface profile oscillate.

\section{ACKNOWLEDGMENT}

D.J.S. gratefully acknowledge the support of the Division of Materials Science of the Office of Basic Energy Sciences of the U.S. Department of Energy Grant No. FG0288ER-45367 under whose auspices this work was performed.

\section{APPENDIX}

In this Appendix, we analyze the state of stress at an interface, as described above. Consider a semi-infinite elastic body $(y>0)$, the surface of which has a periodic normal elastic displacement

$$
u_{B}=U_{B} \sin k x .
$$

Assuming plane-strain conditions, the stresses can be described in terms of the biharmonic Airy stress function $\Psi(x, y)$ :

$$
\begin{aligned}
& \sigma_{x x}=\frac{\partial^{2} \Psi}{\partial y^{2}} ; \quad \sigma_{y y}=-\frac{\partial^{2} \Psi}{\partial x^{2}} ; \quad \sigma_{x y}=\frac{\partial^{2} \Psi}{\partial x \partial y} ; \\
& \sigma_{z z}=\nu\left(\sigma_{x x}+\sigma_{y y}\right)
\end{aligned}
$$

where $\nu$ is the Poisson ratio.

Since the surface elastic displacement has the form indicated in Eq. (A1), we consider a solution to the biharmonic equation of the form $(y>0)$

$$
\Psi=(a+b y) e^{-k y} \sin k x
$$

where $a$ and $b$ are constants. Substituting Eq. (A3) into Eq. (A2) yields

$$
\sigma_{y y}=-k^{2}(a+b y) e^{-k y} \sin k x
$$

and the other components of the stress tensor have similar forms. Using Hooke's law, the strain tensor can be expressed in terms of the stress tensor

$$
\epsilon_{y y}=\frac{1+\nu}{E}\left[-k^{2}(a+b y)+2 k \nu b\right] e^{-k y} \sin k x
$$

where $E$ is Young's modulus. Assuming that the interface slips, we apply the boundary condition $\sigma_{x y}=0$ at the interface $(y=0)$ and find that $b=k a$. Inserting this result into Eq. (A5) yields

$$
\epsilon_{y y}=-\frac{(1+\nu)}{E}[1+k y-2 \nu] e^{-k y} \sigma
$$

where

$$
\sigma \equiv-\sigma_{y y}(y=0)=a k^{2} \sin k x .
$$

The strain $\epsilon_{y y}$ is equal to $\partial u / \partial y$, thus, the elastic displacement $u_{B}$ at the interface can be found from

$$
u_{B}=-\int_{0}^{\infty} \epsilon_{y y} d y=\frac{2\left(1-\nu^{2}\right)}{E} \sigma .
$$

Equation (A8) provides the link between the stress and displacement at the boundary in each phase. Therefore, we can write

$u_{\eta}=\frac{\sigma}{k G_{\eta}} \quad$ where $\quad G_{\eta}=\left[\frac{E}{\left(1-\nu^{2}\right)}\right]_{\eta} ; \quad \eta=A, B$.

The total elastic displacement at the interface $u$ is the sum of $u_{A}$ and $u_{B}$. Employing Eq. (A9), we obtain

$$
\sigma=k G u \quad \text { where } \quad G=\frac{2 G_{A} G_{B}}{\left(G_{A}+G_{B}\right)} .
$$

As follows from Eq. (A9)

$$
\xi \equiv \frac{u_{B}}{u_{A}+u_{B}}=\frac{G_{A}}{G_{A}+G_{B}} .
$$

\footnotetext{
${ }^{1}$ W. W. Mullins and R. F. Sekerka J. Appl. Phys. 35, 444 (1964).

${ }^{2}$ C. Wagner J. Electrochem. Soc. 103, 571 (1956).

${ }^{3}$ M. Roberth, W. Pompe, M. Rocstron, and E. Schumman, Acta Metall. Mater. 42, 579 (1994).

${ }^{4}$ M. A. Grinfeld, Dokl. Akad. Nauk SSSR 290, 1358 (1986); Sov. Phys. Dokl. 31, 831 (1986).

${ }^{5}$ D. J. Srolovitz, Acta Metall. 37, 621 (1988).
} 
${ }^{6}$ N. Junqua and J. Grilhe, Phil. Mag. Lett. 69, 61 (1994).

${ }^{7}$ D. Watkins and H. R. Pielhler, Metall. Trans. A 23, 2669 (1992).

${ }^{8}$ S. Schimschal-Thölke, H. Schmalzried, and M. Martin, Bsr. Bunsenges.

Phys. Chem. 99, 1 (1995).
${ }^{9}$ L. Klinger and L. Levin, J. Appl. Phys. 78, 1669 (1995).

${ }^{10}$ I. A. Blech, J. Appl. Phys. 47, 1203 (1977).

${ }^{11}$ W. W. Mullins, J. Appl. Phys. 28, 333 (1957).

${ }^{12}$ L. Klinger and D. J. Srolovitz (unpublished). 